Meta

Journal des tradlucteurs

Translators' Journal

\title{
Le petit prince en arabe dialectal tunisien
}

\section{Salah Mejri}

Volume 45, numéro 3, septembre 2000

La traduction dans le monde arabe

URI : https://id.erudit.org/iderudit/003615ar

DOI : https://doi.org/10.7202/003615ar

Aller au sommaire du numéro

Éditeur(s)

Les Presses de l'Université de Montréal

ISSN

0026-0452 (imprimé)

1492-1421 (numérique)

Découvrir la revue

Citer cet article

Mejri, S. (2000). Le petit prince en arabe dialectal tunisien. Meta, 45(3), 564-565.

https://doi.org/10.7202/003615ar d'utilisation que vous pouvez consulter en ligne.

https://apropos.erudit.org/fr/usagers/politique-dutilisation/ 


\section{BLOC-NOTES}

\section{Le petit prince en arabe dialectal tunisien}

À notre connaissance, c'est la première fois qu'un texte littéraire de cette qualité est traduit et publié intégralement en tunisien. Ce fait mérite d'être souligné pour diverses raisons:

- il rompt avec les habitudes ancestrales qui ont toujours respecté les différences hiérarchiques entre les langues en situation de diglossie (littéral/ dialectal);

- il traduit une attitude positive vis-à-vis du dialectal puisque le texte traduit n'est pas du type informatif ou strictement fonctionnel mais, au contraire, d'une grande qualité littéraire;

- il constitue objectivement un événement linguistique et éditorial important dans une situation où le dialectal, même s'il connaît une production littéraire orale propre (surtout poétique), n’a jamais prétendu à un tel statut.

C'est ce dernier point qui nous intéresse le plus, parce qu'il nous offre l'occasion de soulever un certain nombre de problèmes linguistiques qui ne manquent pas d'intérêt.

\section{Le dialectal et l'écrit}

Indépendamment de l'attitude qu'on pourrait avoir à l'égard des dialectes, un fait est certain: il s'agit d'un idiome pratiqué spontanément et quotidiennement par la communauté, c'est-à-dire un outil participant à la structuration de l'affectivité des locuteurs. Cela signifie qu'on ne peut en aucune façon lui dénier le droit et l'aptitude à l'expression littéraire. La production littéraire dialectale est toujours là pour convaincre les irréductibles, mais cette production demeure foncièrement d'une nature orale, prenant la forme de poèmes lus publiquement ou de contes racontés dans des cadres précis. Il est vraiment rare d'avoir des documents écrits édités en vue d'être lus à grande échelle. L'un des rares domaines où le dialectal tunisien connaît un statut écrit demeure la publicité, portant sur des produits tunisiens ou étrangers.

Il s'agit donc d'une langue non écrite qui, malgré son caractère arabe indéniable, ne dispose pas d'une orthographe normalisée, ce qui pose des difficultés à la lecture des textes écrits en dialectal. Et puisque l'écrit se fait nécessairement en caractères arabes, un texte dialectal écrit implique un certain nombre d'interférences qui nuisent au décryptage des graphèmes, parmi lesquelles nous retenons:
- La dimension idéogrammique (ou logogrammique) de l'orthographe qui fait que les mots sont appréhendés dans leur configuration générale. Cette dimension concerne surtout les mots les plus fréquents, c'est-à-dire les outils grammaticaux (pronoms, prépositions, conjonctions, etc.). Or, le texte écrit s'adresse normalement aux lettrés qui ont l'habitude des textes en arabe classique (ou standard). Le résultat est un décalage entre une représentation conditionnée par le standard et une orthographe qui rend compte du dialectal. Ce décalage perturbe la lecture et, par conséquent, porte atteinte à l'intégrité du message et à sa spécificité.

- L'absence de marques syntaxiques (les flexions) qui servent de repères dans l'arabe classique pour marquer les différents cas, ce qui peut entraîner des confusions à la lecture du texte dialectal écrit, qui substitue aux marques casuelles la contrainte de l'ordre des mots et celle du recours aux joncteurs syntaxiques. Cela a pour conséquence de faire que certains passages où ne figurent pas les spécificités syntaxiques du dialectal se lisent à la manière de l'arabe standard.

- L'absence de voyellation, presque systématique dans les publications arabes, réduisant les mots à leur structure consonantique enrichie des marques des voyelles longues, ce qui favorise les lectures ambivalentes renvoyant aux deux niveaux de la hiérarchie linguistique.

C'est peut-être pour ces raisons que l'auteur a éprouvé le besoin d'ajouter de temps en temps des marques de voyellation, dont le but n'est pas du tout le marquage casuel mais essentiellement celui de l'appartenance dialectale (cf. le titre en arabe qui, sans les marques de la voyelle [a] et de la gémination de [j], serait lu en littéral: [sүajjar], "petit », d'autant plus que [?al?am:r] : «le prince» est identique dans les deux niveaux de langue).

Les marques du dialectal sont loin d'être réduites à ces repères graphiques: elles les dépassent pour englober des spécificités morphologiques, syntaxiques et lexicales.

\section{Certaines spécificités du dialectal tunisien}

L'un des apports linguistiques indéniables de cette traduction, c'est la conservation, grâce à l'écrit, d'un état du dialectal tunisien, qui n'a pas bénéficié jusque-là, pour des raisons idéologiques, de descriptions systématiques, si l'on met de côté les quelques tentatives réduites à des lexiques ou à des descriptions sommaires conçues à des fins didacti- 
ques. Partant de ce texte, il nous est possible de relever un nombre de spécificités linguistiques qui rompent avec celles du littéral.

Sur le plan morpho-syntaxique, il y a lieu de constater un certain nombre de ruptures : la disparition du duel en tant qu'expression de la catégorie du nombre ayant des spécificités morpho-syntaxiques, celle des marques casuelles, la création d'une série de particules propres touchant des aspects aussi variés que la négation, l'interrogation, les démonstratifs, les personnels, etc.

L'importance de l'emprunt et des expressions idiomatiques: dans le dialectal, l'emprunt n'est pas senti comme une menace puisque cette langue n'est pas normalisée. Tout terme étranger répondant à un besoin d'expression ou de dénomination est tout naturellement intégré dans le lexique. Dans cette traduction, nombreux sont les mots ou expressions d'origine française: [kri:zrumati:zm], «crise de rhumatisme» (p. 61); [ minijja], «cheminée» (p. 43); [tri:si :ti], «électricité» (p. 81); [tnerviz], «s'énerver» (p. 23); [tuht?ampan], «tomber en panne» (p. 109); [kurande:r], «courant d'air» (p. 39); etc.

Si le grand pouvoir d'intégration des emprunts témoigne de la vitalité du lexique du dialectal, l'importance de l'idiomaticité fournit la preuve que le dialectal n'est pas un simple outil véhiculaire mais qu'il est le support d'une culture propre et d'une vision du monde qui transparaissent à travers toutes sortes de séquences figées: les expressions, les proverbes, les formules de salutation, etc. La présence très saillante de ces idiotismes reflètent en fait le souci du traducteur de produire un texte portant réellement l'empreinte du dialectal.

Spécificités syntaxiques, morpho-syntaxiques et lexicales concourent à faire de cette traduction un texte-échantillon qui pourrait servir de point de départ à l'étude du dialectal tunisien.

Salah MejRi 